\title{
Aspects of the Coriolis Interaction in ${ }^{235} \mathbf{U}$
}

\author{
D. Ward*, R.M. Clark*, M. Cromaz*, M.A. Deleplanque*, R.M. Diamond*, \\ P. Fallon*, G.J. Lane ${ }^{* \dagger}$, I.Y. Lee*, A. Görgen ${ }^{* * *}$, A.O. Macchiavelli*, F.S. Stephens*, \\ C.E. Svensson ${ }^{* \ddagger}$, K. Vetter ${ }^{* \S}$, D. Cline ${ }^{\mathbb{I I}}$, A.B. Hayes ${ }^{\mathbb{T}}$, R. Teng ${ }^{\mathbb{I I}}$, C.-Y. Wu ${ }^{\mathbb{U}}$ and \\ T. Nakatsukasall \\ *Lawrence Berkeley National Laboratory, Berkeley, CA 94720, USA \\ ${ }^{\dagger}$ Australian National University, Canberra, Australia \\ ${ }^{* *}$ CEA Saclay, F-91191 Gif-sur-Yvette, France \\ ${ }^{\ddagger}$ University of Guelph, Guelph, Ontario N1G2W1, Canada \\ ${ }^{\S}$ Lawrence Livermore National Laboratory, Livermore, CA 94550, USA \\ "I University of Rochester, Rochester, NY 14627, USA \\ UUniversity of Tsukuba, Tsukuba 305-8571, Japan
}

\begin{abstract}
We have performed three separate experiments at LBNL's 88-Inch Cyclotron over a period of several years in which ${ }^{235} \mathrm{U}$ (thick target) was Coulomb-excited. It involved stand-alone experiments with Gammasphere and with the 8PI Spectrometer using ${ }^{136} \mathrm{Xe}$ beams at $720 \mathrm{MeV}$, and a CHICO-Gammasphere experiment with an ${ }^{40} \mathrm{Ar}$ beam at $180 \mathrm{MeV}$. In addition to extending the known negative-parity bands to high spin, we have assigned levels in some seven positive-parity bands which are in some cases ( e.g. [631]1/2, [624]7/2 and [622]5/2) strongly populated by E3 excitation. The CHICO data has been analyzed to extract E2 and E3 matrix elements from the observed yields. Additionally, many E1 and M1 matrix elements could be extracted from the $\gamma$-ray branching ratios.
\end{abstract}

\section{INTRODUCTION}

In this paper our emphasis is on the damping of the Coriolis interaction amongst the members of the $j_{15 / 2}$ multiplet. In their paper of 1968, Stephens et al. [1] recognized the unique role of ${ }^{235} \mathrm{U}$ as a laboratory for the study of Coriolis effects in nuclei. First, Coriolis matrix elements increase with increasing $\mathrm{j}$ of the orbitals, and the $j_{15 / 2}$ multiplet is the highest $j$-value attainable in normal nuclei. Second, because the Fermi surface lies close to the middle of the system, near the $\mathrm{K}=7 / 2$ member ([743]7/2 is in fact the ground state), rotational bands on all eight members of the multiplet, $\mathrm{K}=1 / 2$ through $\mathrm{K}=15 / 2$, should lie reasonably low in the spectrum, and in principle be observable. Third, although several nuclei near ${ }^{235} \mathrm{U}$ might satisfy these conditions, ${ }^{235} \mathrm{U}$ and ${ }^{237} \mathrm{Pu}$ are the only cases where a member of the $j_{15 / 2}$ multiplet is the ground state; this property is essential if we are to measure some of the E2 matrix elements between members of the multiplet by Coulomb excitation, and hence determine the mixing amplitudes directly. With all these advantages, it should be possible in ${ }^{235} \mathrm{U}$ to obtain a detailed view of the Coriolis interaction.

\section{EXPERIMENT}

We note that $\gamma-\gamma$ coincidence techniques work poorly at low spins in ${ }^{235} \mathrm{U}$; states of interest decay by one-step transitions to low-lying states of the ground band whose subsequent decays are largely converted. But fusion reactions with light contaminants in the target produce floods of cascades with high $\gamma$-ray multiplicity. Therefore, high-fold $\gamma$ coincidences make the interesting transitions invisible, whilst enhancing the background. In both the Gammasphere and 8PI Spectrometer experiments, the data were replayed so as to retain low coincidence-fold data. The resulting trigger bias cleaned-up the spectra but made it too difficult to extract reliable and quantitative relative cross-sections in Coulomb excitation. These problems were solved in a final experiment with the CHICO array [2] in which ions 
scattered backward of 90 degrees were registered in coincidence with one or more $\gamma$-rays detected in Gammasphere. The ${ }^{235} \mathrm{U}$ target was thick enough to stop the $180 \mathrm{MeV}$ incident ${ }^{40} \mathrm{Ar}$ beam. A portion of the spectrum is shown in Fig. 1. The full spectrum contains approximately 300 resolvable lines in the energy range $150-1700 \mathrm{keV}$, most of which we assigned to ${ }^{235} \mathrm{U}$.

\section{COMPARISON OF MEASURED YIELDS WITH COULOMB EXCITATION CALCULATIONS}

In these experiments matrix elements were extracted by normalizing to the relative yields of the ground-state band for which we take $Q_{0}=9.75 \mathrm{eb}$ from the literature. The E2- and E3-matrix elements needed to calculate the cross-sections are as follows:- within a given band we assume that the reduced E2 transition probabilities are given by this common $Q_{0}$-value. Reduced transition probabilities between the ground-state band and the excited bands were assumed to follow Alaga's rules:-

$$
B\left(E \lambda ; J_{i} \rightarrow J_{f}\right)=((2 \lambda+1) / 16 \pi) e^{2} \quad Q_{t}^{2}(\lambda)<J_{i} K \lambda 0 \mid J_{f} K>^{2}
$$

where $Q_{t}(\lambda)$ plays an analogous role to the intrinsic moments $Q_{0}(\lambda)$

We neglect E1 excitations between the ground-state band and the positive-parity bands, and neglect all matrix elements between states of two different bands except where one band is the ground state band.

Some model dependence is inevitable since the number of unknown matrix elements entering into the Coulomb excitation process is very large. Coriolis mixing of the kind we are most interested in is of the type $\Delta \mathrm{K}=1$, for example between the ground state, [743]7/2, and the [752]5/2 and [734]9/2 bands. Bohr and Mottelson [3] have shown that the intrinsic E2 matrix elements (i.e. between the Nilsson states) are renormalized by the mixing and the form of eq. 1 remains valid.

For the gamma-vibrational bands, $\Delta \mathrm{K}=2$ Coriolis mixing can be treated in lowest order by

$$
M\left(E 2 ; J_{f} K_{f} \rightarrow J_{i} K_{i}\right)=\left(2 J_{i}+1\right)^{1 / 2}<J_{i} K_{i} 22 \mid J_{f} K_{f}>\left(M_{1}+M_{2}\left(J_{f}\left(J_{f}+1\right)-J_{i}\left(J_{i}+1\right)\right)\right)
$$

where $K_{f}=K_{i}+2$. (cf. Bohr and Mottelson [4])

To compare with experiment we need to run the Coulomb excitation code over the active scattering angles and bombarding energies of events seen by the CHICO detectors. The results are summarized in Table 1. Fig. 2 shows the result for the $[624] 7 / 2$ band.

\section{CORIOLIS EFFECTS IN THE $\mathbf{J}_{15 / 2}$ MULTIPLET}

With the parameter set derived in [1], we find that an exact diagonalization of the Coriolis interactions within the $j_{15 / 2}$ multiplet reproduces the earlier result shown in Fig. 3 as the filled points. This fit involves the attenuation of the $<5 / 2\left|j_{+}\right| 3 / 2>$ and $<7 / 2\left|j_{+}\right| 5 / 2>$ Coriolis matrix elements calculated in a Nilsson model by about a factor of two: a result extensively discussed by Stephens et al. [1] and by Bohr and Mottelson [3]. With the new data shown as open points, we see that with increasing spin, there is a general departure from the predictions of the earlier best parameters.

The present measurement of $Q_{t}(\lambda=2)$ for the [752]5/2 and [734]9/2 bands are smaller than those obtained in ref. [1] and imply less mixing than the amplitudes derived from the energies alone. If we assume that the intrinsic $Q_{t}(\lambda=2)$ are zero, then in lowest order:

$$
Q_{t}(\lambda=2)=6^{1 / 2} \quad \alpha \quad Q_{0}
$$

where $\alpha$ is a reduced mixing amplitude which is related to the observed amplitudes $\varepsilon(\mathrm{I})$ by:

$$
\varepsilon(I)=[(I \mp K)(I \pm K+1)]^{1 / 2} \quad \alpha
$$

Comparing experiment and theory, we find that the Coriolis matrix elements $\left\langle 9 / 2\left|j_{+}\right| 7 / 2\right\rangle$ and $\left\langle 7 / 2\left|j_{+}\right| 5 / 2\right\rangle$ are approximately $1 / 3$ of the full Nilsson value. As discussed in [1], pairing will reduce the Coriolis matrix elements from the ideal value, but this is significant only for states on opposite sides of the Fermi surface, and even then it can only explain about $10-15 \%$ of the effect in ${ }^{235} \mathrm{U}$.

A plausible explanation to us is that the $j_{15 / 2}$ multiplet has mixed with other Nilsson states and consequently has a smaller $\mathrm{j}$-value than that calculated in a simple model. Since the $j_{15 / 2}$ multiplet largely intrudes into positive-parity 
TABLE 1. Rotational Bands Observed in these Experiments

\begin{tabular}{|c|c|c|c|c|}
\hline $\begin{array}{c}\text { Spherical } \\
\text { Shell }\end{array}$ & $\begin{array}{c}\text { Nilsson } \\
\text { Orbital }\end{array}$ & $\mathrm{K}$ & $\begin{array}{c}\text { Max Spin } \\
\text { Observed }\end{array}$ & $\begin{array}{c}\text { Transition Moment } \\
\mathrm{Q}_{t}(\lambda=3) \mathrm{e}^{3} \mathrm{fm}^{3}\end{array}$ \\
\hline $\mathrm{i}_{11 / 2}$ & {$[631]$} & $3 / 2^{+}$ & $43 / 2^{+}$ & 0.79 \\
& {$[622]$} & $5 / 2^{+}$ & $49 / 2^{+}$ & 1.05 \\
& {$[613]$} & $7 / 2^{+}$ & $13 / 2^{+}$ & 0.61 \\
$\mathrm{~g}_{9 / 2}$ & {$[633]$} & $5 / 2^{+}$ & $13 / 2^{+}$ & 0.80 \\
& {$[624]$} & $7 / 2^{+}$ & $15 / 2^{+}$ & 1.03 \\
& {$[615]$} & $92^{+}$ & $15 / 2^{+}$ & 0.70 \\
$\mathrm{~d}_{5 / 2}$ & {$[631]$} & $1 / 2^{+}$ & $53 / 2^{+}$ & 1.75 \\
& & & & \\
\hline \multirow{3}{*}{$\mathrm{j}_{15 / 2}$} & & & & $\mathrm{Transition} \mathrm{Moment}^{2}$ \\
& & & & $\mathrm{Q}_{t}(\lambda=2) \mathrm{e} 10^{2} \mathrm{fm}^{2}$ \\
& {$[752]$} & $5 / 2^{-}$ & $41 / 2^{-}$ & 0.50 \\
& {$[743]$} & $7 / 2^{-}$ & $57 / 2^{-}$ & 9.75 \\
$($ Gamma vibration) & & $9 / 2^{-}$ & $35 / 2^{-}$ & 0.38 \\
& & $3 / 2^{-}$ & $31 / 2^{-}$ & 0.44 \\
& & $11 / 2^{-}$ & $23 / 2^{-}$ & $\mathrm{M}_{1} / \mathrm{M}_{2}=0.031$ \\
& & & & 1.39 \\
& & & & $\mathrm{M}_{1} / \mathrm{M}_{2}=0.027$ \\
\hline
\end{tabular}

levels, this mixing can only be through octupole correlations. We have evidence that there are indeed collective E3 excitations in ${ }^{235} \mathrm{U}$. There are orbitals nearby, particularly the $g_{9 / 2}$ multiplet, that give rise to such octupole correlations. We have begun a program to calculate octupole mixing in the Random Phase Approximation (RPA). Gareev et al. generally reported similar results [5]. Some preliminary findings are that the [624]7/2 state contains an admixture of the [743]7/2 coupled to the $\mathrm{K}=0$ phonon with an amplitude in the range $0.36-0.53$, which accounts for the strong E3 excitation of the [624]7/2 band. The [622]5/2 state is mixed into the [734]9/2 via the $\mathrm{K}=2$ phonon with an amplitude in the range 0.4-0.53. This could account for the strong attenuation of the Coriolis matrix element $\left\langle 9 / 2\left|j_{+}\right| 7 / 2\right\rangle$. The results are generally in line with the experiments; however, the calculations say that the [631]1/2 is almost a pure Nilsson state, whereas we find that band to be the most collective of the E3 excitations.

\section{CONCLUSIONS}

We have pointed out that a large component of the observed Coriolis attenuation in ${ }^{235} \mathrm{U}$ at low spin may be due to E3 correlations. Preliminary calculations with the RPA give support to this idea, but do not give a completely satisfactory explanation so far. Other avenues are still to be explored. For example, the calculations predict that the [631]1/2 band has pure quasi-particle character, but we find it to be strongly excited by E3 Coulomb excitation. Within the $j_{15 / 2}$ multiplet, the calculations say that the [743]7/2 and [752]5/2 bands are both relatively unmixed with octupole phonons, but that the [734]9/2 is strongly mixed. Although we have not included E2 collectivity, we note that Gareev et al. [5] find strong mixing of E2 phonons into the [770]1/2 and [761]3/2 levels. Thus we may be uncovering a rather complex situation where many effects are combining to produce the Coriolis attenuation at low spin.

At high spin, even the empirically attenuated Coriolis matrix elements produce too much effect. This may be due to inadequacies of the particle-rotor model, which can not take into account 3-quasi-particle effects. Also it is clear that the gamma-vibrational bands $(\mathrm{K}=3 / 2$ and $\mathrm{K}=11 / 2)$ participate in the interactions of the $j_{15 / 2}$ multiplet and must be taken into account. 


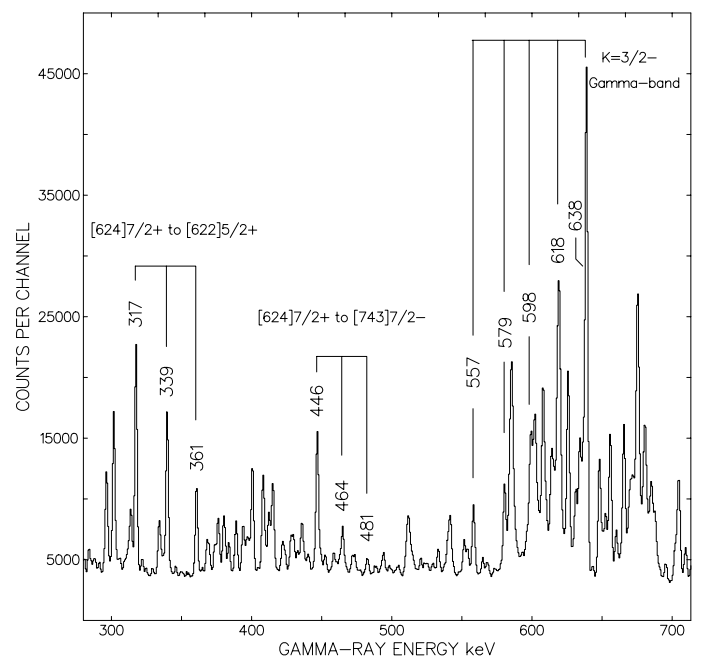

FIGURE 1. A portion of the $\gamma$-ray spectrum in coincidence with beam particles detected in CHICO. Note the strength of the E3 excitation to the positive parity [624]7/2 state compared with a collective E2 excitation to the $\gamma$ vibrational band. Almost all of the transitions in this complex spectrum can be assigned to ${ }^{235} \mathrm{U}$.

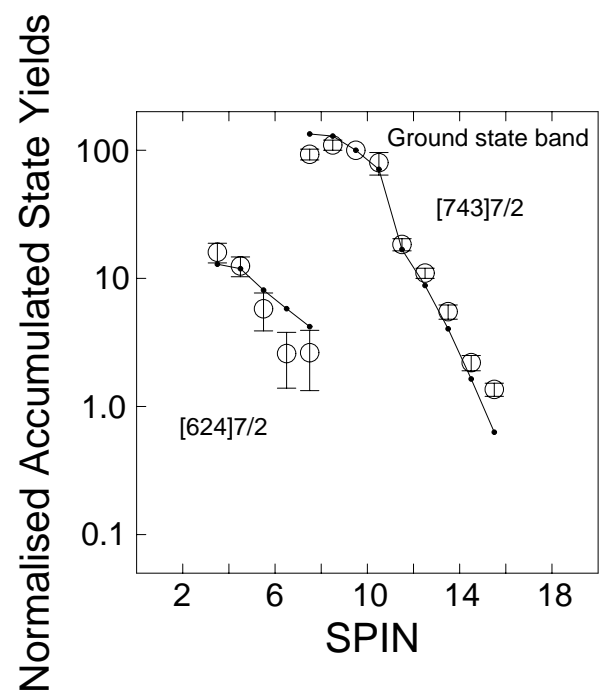

FIGURE 2. Measured and calculated yields for rotational bands in ${ }^{235} \mathrm{U}$. Note that the ground-band values refer only to the E2 gamma-ray branch. The result for the [624]7/2 band is $Q_{t}(\lambda=3)=1.03 \mathrm{e} 10^{3} \mathrm{fm}^{3} \mathrm{~cm}^{3}$. 


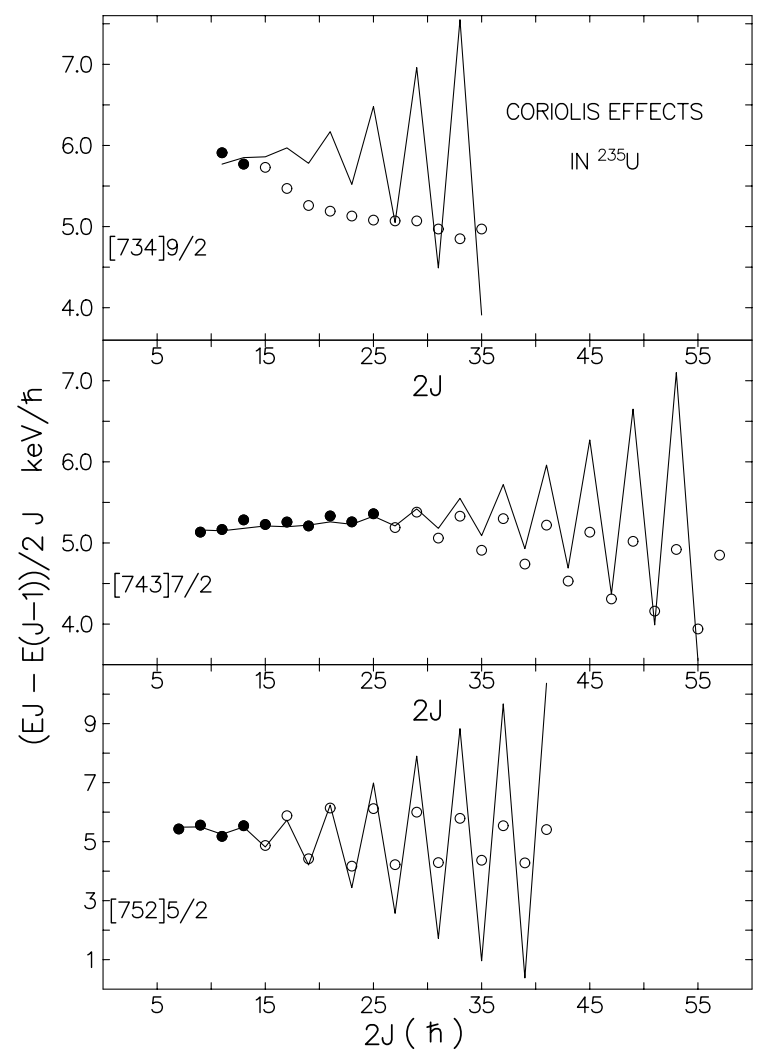

FIGURE 3. The rotational constant calculated for members of the $j_{15 / 2}$ multiplet with the parameters of ref. [1]. The filled points were known and fitted in ref. [1], the open points are from the present data.

\section{ACKNOWLEDGMENTS}

This work was supported by the Director, Office of Energy Research, Division of Nuclear Physics of the Office of High Energy and Nuclear Physics of the U.S. Department of Energy under contract No. DE-AC03-76sf00098.

\section{REFERENCES}

1. $\quad$ F.S. Stephens, M.D. Holtz, R.M. Diamond, and J.O. Newton; Nucl. Phys. A115 (1968) 129

2. M.W.Simon et. al. Nucl. Inst. and Methods A452 (2000) 205

3. $\quad$ A. Bohr and B.M. Mottelson; Nuclear Structure Vol 2, pages 273-284

4. A. Bohr and B.M. Mottelson; Nuclear Structure Vol 2, pages 149-151

5. $\quad$ F.A. Gareev, S.P. Ivanova, A. Malov, and V.G. Soloviev; Nucl. Phys. A171 (1971) 134 